\title{
Electronic Structure of Brain: Structure-Activity Relationships between Electronic Structure and Neurotransmitters Based on Molecular Hardness Concept
}

\author{
Shigeki KoBAYASHI* and Yuki TERAO \\ Department of Analytical Chemistry of Medicines, Showa Pharmaceutical University; 3-3165 Higashi-tamagawagakuen, \\ Machida, Tokyo 194-8543, Japan. \\ Received October 16, 2003; accepted February 3, 2004
}

In order to understand the relation between the electronic structure of neurotransmitters and the brain, a model of the brain based on absolute hardness $(\eta)$ and absolute electronegativity $(\chi)$ is described. It was found that the coordinate $r(\chi, \eta)$ of electronic structures of neurotransmitters obtained using the parameters $\eta$ and $\chi$ can be graphically classified into three groups: catecholamine type (group I), gamma-aminobutanoic acid (GABA) type (group II), and acetylcholine (ACh) type (group III) in the $\eta-\chi$ diagram. The results suggest that the brainstem and neocortex in the brain are chemically soft and hard, respectively, because they show that the myelinated nerve is chemically soft and the unmyelinated nerve is chemically hard. If one calculates the $r(\chi, \eta)$ to understand which group a drug belongs to, one can predict the target receptors of the drug from the $\eta-\chi$ diagram. Using $\eta-\chi$ maps, one is then able to design medications like antidepressants, tranquilizers, and ACh agonists.

Key words chemical hardness; neurotransmitter; structure-activity relationship; brain; antidepressant; $\eta-\chi$ diagram

Hardness concept-based structure-activity relationships (SARs) of new quinolones, dioxins, PCBs, and environmental hormones as one application of biological activity have recently been reported. ${ }^{1,2}$ The method suggests that more toxic isomers of dioxins are chemically soft, whereas less toxic isomers are chemically hard. The antibacterial activity of potent antibacterial drugs like norfloxacin, enoxacin, etc. are controlled by absolute electronegativity $(\chi)$, not by absolute hardness $(\eta)^{2-6)}$ The $\eta-\chi$ diagrams used in the SAR investigations are composed of a coordinate $\boldsymbol{r}\left((-\partial E / \partial N)_{v(r)}\right.$, $\left.\left.\left(\partial^{2} E / \partial N^{2}\right)_{v(r)}\right)\right)(=r(\chi, \eta))$ of the electronic structure of the drugs and chemicals, where $E$ is the electronic energy of a molecule, $N$ is the number of electrons, and $v(r)$ is the external electrostatic potential. Using two factors, $\chi$ and $\eta$, the $\eta-\chi$ diagram gives the coordinates of an electronic structure as a new way of predicting ligands, agonists, and antagonists of drugs and chemicals. Here the diagram is applied to the central nervous system in the brain.

It has been reported that the potency of activity (molar ratio of equipotency) for the rectus abdominis of frog of acetylcholine $(\mathrm{ACh})$ derivatives increases in the following order: $\mathrm{AcOCH}_{2} \mathrm{CH}_{2} \mathrm{~N}^{+}(\mathrm{Et})_{3}<\mathrm{AcOCH}_{2} \mathrm{CH}_{2} \mathrm{~N}^{+}(\mathrm{Me})(\mathrm{Et})_{2}<$ $\mathrm{AcOCH}_{2} \mathrm{CH}_{2} \mathrm{~N}^{+}(\mathrm{Me})_{2}(\mathrm{Et})<\mathrm{AcOCH}_{2} \mathrm{CH}_{2} \mathrm{~N}^{+}(\mathrm{Me})_{3}{ }^{7}{ }^{7}$ This suggests that the size of the alkyl group binding to the tertiary $N$ atom is attributable to the order of the activity. Although it is generally recognized that the binding affinity of a drug is affected by chemical properties like steric, hydrophobic, and hydrogen-bonding at the surface of the receptor, we show that the strength of the activity of ACh derivatives is inversely proportional to the global hardness $\eta$ in the coordinate $r(\chi, \eta)$ of the electronic structure. This supports that the coordinate of the neurotransmitters also plays an important role as a measure of the binding force needed to form a molecular complex between the chemical and target receptor. The electronic states of neurotransmitters like ACh, dopamine, serotonin and noradrenaline were calculated at the Hartree-Fock level using a $6-31 \mathrm{G}^{* *}$ basis set. A $\eta-\chi$ dia- gram of neurotransmitters plotted using the electronic structure's coordinate $\boldsymbol{r}(\chi, \eta)$ is shown here. It was found that the electronic structures of neurotransmitters can be classified into three groups: catecholamine type (group I), gammaaminobutanoic acid (GABA) type (group II), and acetylcholine type (group III).

What kind of electronic structure does the central nervous system have? This paper describes the electronic structure of the central nervous system in the brain using $\eta-\chi$ diagrams for neurotransmitters. The results show that myelinated nerve fiber is chemically soft and the unmyelinated nerve fiber is chemically hard. The brainstem is chemically soft. If we calculate the coordinate $r(\chi, \eta)$ of each chemical structure to understand to which group a target drug belongs, we can predict on which nerve fiber the target drug acts on. Furthermore, the $\eta-\chi$ diagram can be used to determine to which groups the target drugs or chemicals belong. The results could be used for the development of agonists and antagonists. According to our results, the antidepressants-fluoxetine and milnacipran ${ }^{8}$ - are distributed in group I in the $\eta-\chi$ diagram. The coordinates of their electronic structures are similar to those of catecholamine and serotonin. The method may thus be useful for designing antidepressants, tranquilizers, ACh agonists, etc. Essential for the functional expression of a drug is that it have an electronic structure coordinate $r(\chi, \eta)$ similar to the ligand. Thus we describe the first qualitative analysis of the electronic structure of the brain based on the coordinates of a series of neurotransmitters.

\section{Experimental}

Geometries In this study, the geometries of neurotransmitters optimized structures obtained from analysis using the "conformer distribution" were optimized with Hartree-Fock ab initio calculations using a $6-31 \mathrm{G}^{* *}$ basis set. ${ }^{9)}$ The chemical structures and resultant structures, optimized bond lengths and electron density, are shown in Figs. 1 and 2. For neurotransmitters of amino acid types like GABA, Glu, Asp, and taurine, we obtained the electronic structure and optimized geometry for the zwitterionic form $\mathrm{H}_{3} \mathrm{~N}^{+}-\mathrm{CH}(\mathrm{R})-\mathrm{COO}^{-}$.

Absolute Hardness and Electronegativity We obtained the highest oc- 


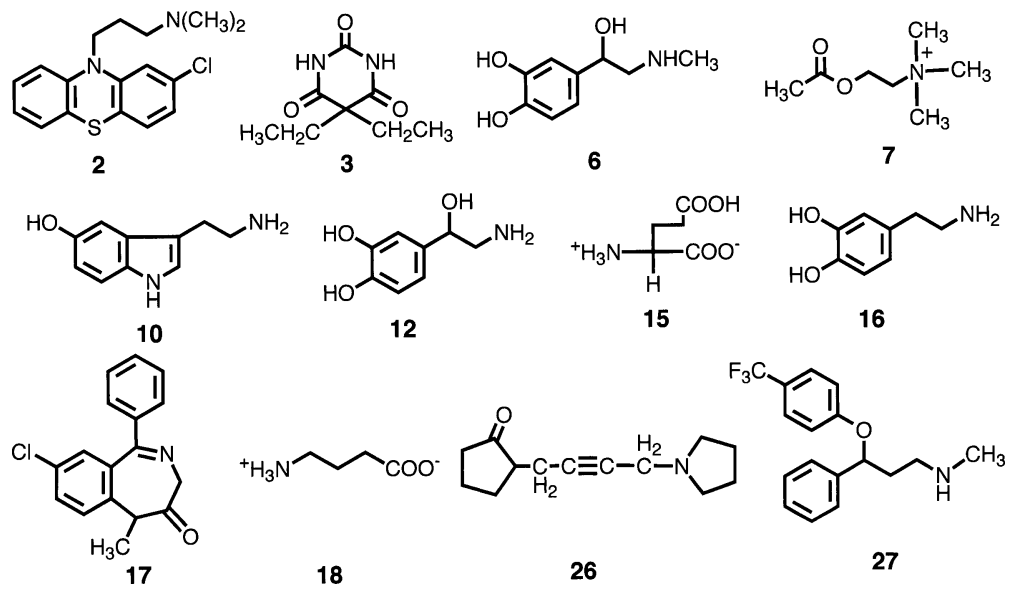

Fig. 1. Chemical Structures of Several Neurotransmitters

Numbers show the compounds listed in Table 1.

cupied (homo) and lowest unoccupied (lumo) molecular orbital energies $(\mathrm{eV}), \varepsilon_{\text {homo }}$ and $\varepsilon_{\text {lumo }}$, respectively, of the targets with the $6-31 \mathrm{G}^{* *}$ basis set. From $\varepsilon_{\text {homo }}$ and $\varepsilon_{\text {lumo }}$ orbital energies, the values of absolute hardness $(\eta)$ and absolute electronegativity $(\chi)$ were approximated by Eqs. 1 and 2, as defined by Parr and Pearson, ${ }^{10)}$

$$
\begin{aligned}
& \chi=-\mu=-(\partial E / \partial N)_{v(r)}=\left(I_{\mathrm{p}}+E_{\mathrm{a}}\right) / 2 \\
& \eta=1 / 2(\partial \mu / \partial N)_{v(r)}=1 / 2\left(\partial^{2} E / \partial N^{2}\right)_{v(r)}=\left(I_{\mathrm{p}}-E_{\mathrm{a}}\right) / 2
\end{aligned}
$$

where $E$ is the electronic energy of a molecule and $N$ is the number of electrons, and $v(r)$ is the external electrostatic potential. $I_{\mathrm{p}}$ and $E_{\mathrm{a}}$ are the ionization energy and the electron affinity $(\mathrm{eV})$, respectively, and are used to calculate the $\eta$ and $\chi$ values using Eqs. 3 and 4 .

$$
\begin{aligned}
& \chi=-1 / 2\left(\varepsilon_{\text {homo }}+\varepsilon_{\text {lumo }}\right) \\
& \eta=1 / 2\left(\varepsilon_{\text {lumo }}-\varepsilon_{\text {homo }}\right)
\end{aligned}
$$

The soft acids in chemicals will prefer to coordinate with soft bases, whereas hard acids will prefer hard bases (HSAB principle). ${ }^{4)}$

$\eta-\chi$ Activity Diagram We represent the $\eta-\chi$ activity diagram as the coordinate $r(\chi, \eta)$ of electronic structures of neurotransmitters. In the diagram, we used $\chi$ as the abscissa and $\eta$ as the ordinate. Chemical properties of the neurotransmitters can be defined from the coordinate $r(\chi, \eta)$. The $\chi$ of the abscissa and the $\eta$ of the ordinate exhibit the basicity and the polarizability of a given chemical, respectively. Hard chemicals have a large $\eta$ value and soft chemicals have a small $\eta$ value.

\section{Results}

Structure and Chemical Hardness and $\eta-\chi$ Activity Diagram of Central Neurotransmitters The first step is to calculate the chemical hardness $(\eta)$, electronegativity $(\chi)$, and global softness $(S)$ using Eqs. 3-5 for optimized neurotransmitters like acetylcholine (ACh), dopamine, GABA, nicotine and serotonin in the central nervous system. The lowest energy conformation obtained with the conformational search calculation (at the PM3 level) was optimized using the HF/6-31G** basis set. The calculated results are listed in Table 1. Several studies support that the muscarine and nicotine actions of $\mathrm{ACh}$ are related to the conformations of $\mathrm{ACh}$ in the receptors. For example, it is known that $\mathrm{ACh}$ has two, transoid and cisoid, conformers in contact with muscarinic and nicotinic ACh receptors, respectively, and the cisoid conformer is over $2 \mathrm{kcal} / \mathrm{mol}$ more stable than the transoid conformer. ${ }^{11)}$ Good agreement between our HF/6$31 \mathrm{G}^{* *}$-optimized data and several models utilizing NMR and X-ray data ${ }^{12,13)}$ of ACh was found. The structures $7 \mathbf{a}-\mathbf{c}$ in Fig. 2 show the HF/6-31G** optimized conformers of the

\begin{tabular}{|c|c|c|c|}
\hline \multirow{2}{*}{ No. } & \multirow{2}{*}{ Compounds } & \multirow{2}{*}{$\begin{array}{c}\begin{array}{c}\text { Absolute } \\
\text { electronegativity }\end{array} \\
(\chi, \mathrm{eV})^{a)}\end{array}$} & \multirow{2}{*}{$\begin{array}{l}\text { Absolute } \\
\text { hardness }\end{array}$} \\
\hline & & & \\
\hline 1 & Choline & 7.780 & 8.700 \\
\hline 2 & Chlorpromazine & 2.240 & 5.460 \\
\hline 3 & Barbital & 4.295 & 7.555 \\
\hline 4 & Asp & 3.075 & 7.195 \\
\hline 5 & Amphetamine & 2.450 & 6.330 \\
\hline 6 & Adrenaline & 1.980 & 6.040 \\
\hline 7 & Acetylcholine & 7.415 & 8.175 \\
\hline 8 & Thyramine & 2.260 & 6.000 \\
\hline 9 & Taurine & 3.600 & 7.350 \\
\hline 10 & Serotonin & 2.080 & 5.570 \\
\hline 11 & Phenethylamine & 2.455 & 6.335 \\
\hline 12 & Noradrenaline & 2.325 & 6.005 \\
\hline 13 & $m$-Thyramine & 2.285 & 6.125 \\
\hline 14 & Haloperidol & 3.175 & 5.615 \\
\hline 15 & Glu & 2.845 & 7.245 \\
\hline 16 & Dopamine & 2.060 & 6.010 \\
\hline 17 & Diazepam & 3.365 & 5.545 \\
\hline 18 & GABA & 2.965 & 8.165 \\
\hline 19 & Guanidiyltaurine & 3.795 & 7.425 \\
\hline 20 & Nicotine & 6.700 & 6.240 \\
\hline 21 & Muscarine & 6.715 & 7.915 \\
\hline 25 & Imipramine & 2.070 & 5.840 \\
\hline 26 & Oxotremorine & 6.110 & 7.360 \\
\hline 27 & Fluoxetine & 2.740 & 6.140 \\
\hline 28 & Milnacipran & 2.490 & 6.260 \\
\hline
\end{tabular}
cisoids $(\mathbf{7} \mathbf{a}, \mathbf{b})$ and transoid $(\mathbf{7 c})$ of $\mathrm{ACh}$. The cisoid $\mathbf{7 a}$ is
Table 1. Calculated Absolute Hardness $(\eta)$ and Electronegativity $(\chi)$ of Optimized Neurotransmitters

a) at ab initio $\mathrm{HF} / 6-31 \mathrm{G}^{* *}$ level.

about $8.8 \mathrm{kcal} / \mathrm{mol}$ more stable than the other cisoid $7 \mathbf{b}$. The distances $\mathrm{C}=\underline{\mathrm{O}} \ldots \underline{\mathrm{N}}\left(\mathrm{CH}_{3}\right)_{3}$ and $\underline{\mathrm{O}} \ldots \underline{\mathrm{N}}\left(\mathrm{CH}_{3}\right)_{3}$ in 7a obtained from $\mathrm{HF}$ calculation using the $6-31 \mathrm{G}^{* *}$ basis set are about 4.969 and $3.030 \AA$, respectively. The results indicate that the cisoid $7 \mathbf{a}$ is a conformation used to express the muscarinelike action of $\mathrm{ACh} .{ }^{14)}$ The dihedral angles $(\phi), \mathrm{O}-\mathrm{C}(2) \mathrm{H}_{2}-$ $\mathrm{C}(1) \mathrm{H}_{2}-\mathrm{N}$, in the isomers $7 \mathbf{a}$ and $7 \mathbf{b}$ are about -62.4 and $-58.2^{\circ}$, respectively. The $\phi$ of transoid $7 \mathbf{c}$ is $156.2^{\circ}$, and the distance $(3.820 \AA)$ of $\mathrm{C}=\underline{\mathrm{O}} \ldots \underline{\mathrm{N}}\left(\mathrm{CH}_{3}\right)_{3}$ is indicative of the geometry of nicotinic ACh.

Each conformer is also represented by the Newman projection, as shown in Fig. 2. Clearly, the geometry of cisoid 7a differs from that of transoid 7c. However, the result indi- 
(a)

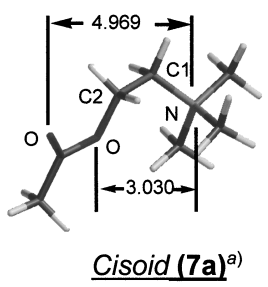

(b)

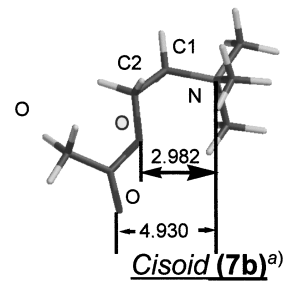

(c)

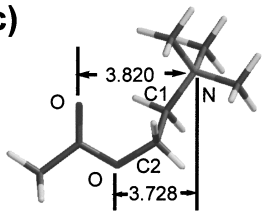

Transoid (7c) ${ }^{a)}$
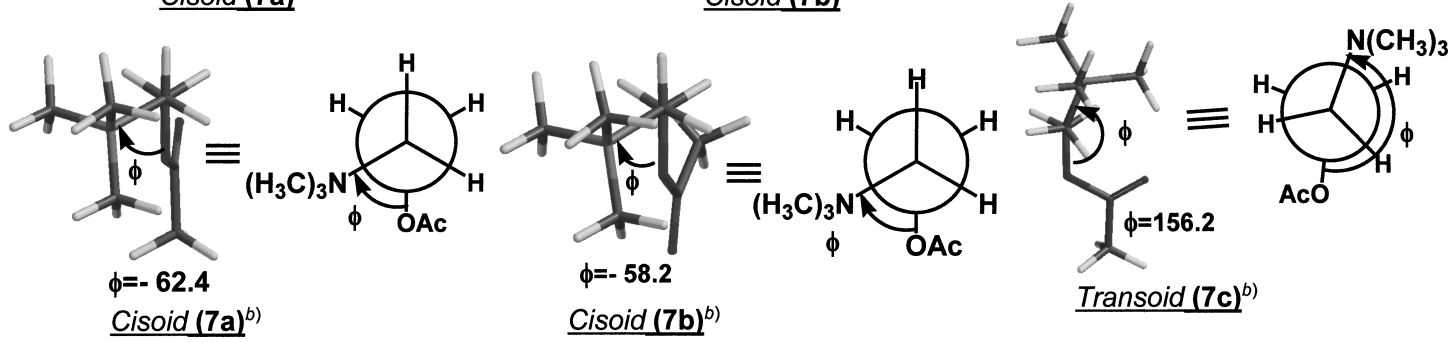

Transoid $(\mathbf{7 c})^{b)}$

Fig. 2. Tube Representation of Optimized Conformations, Cisoid and Transoid, of Acetylcholine Obtained from HF/6-31G** Calculations

a) Conformers $\mathbf{7 a}, \mathbf{b}$ and $\mathbf{7 c}$ show cisoid and transoid conformations of $\mathrm{ACh}$, respectively. The numerical values are the distance between the ammonium $\mathrm{N}^{+}$and oxygen $\mathrm{O}_{\text {, and }}$ the $\phi$ is a dihedral angle of the $\mathrm{N}^{+}-\mathrm{C}_{1}-\mathrm{C}_{2}-\mathrm{O}$ in $\mathrm{ACh}$. Each value is an angstrom and a degree unit, respectively. $b$ ) The figure shown is Newman projection.

cates that acetylcholine is conformationally free in cisoid $\rightleftarrows$ tansoid equilibrium since conformer $7 \mathbf{c}$ is only $0.1 \mathrm{kcal} / \mathrm{mol}$ more stable than conformer $\mathbf{7 a}$ according to $\mathrm{HF} / 6-31 \mathrm{G}^{* *}$ calculation. As the difference of nicotinic and muscarinic action can not be discussed by the total energy change, we used the coordinate $r(\chi, \eta)$ of the electronic structure. The coordinate $\boldsymbol{r}(\chi, \eta)$ of ACh in the muscarine-like action is $\boldsymbol{r}(7.415$, $8.175)$, and the value of $\mathrm{ACh}$ in the nicotine-like action is about $\boldsymbol{r}(7.255,8.055)$. We showed that the muscarine-like and nicotine-like actions of ACh are important to the electronic structure as well as the most stable conformation (see Fig. 3). For these reasons, we used $\boldsymbol{r}(7.415,8.175)$ as the value of $\mathrm{ACh}$ in the plotting of the $\eta-\chi$ diagram.

To elucidate the relationship between the receptor binding and electronic structure of central neurotransmitters, we present a $\eta-\chi$ activity diagram as a coordinate of the HF/6$31 \mathrm{G}^{* *}$ calculated electronic structures for central neurotransmitters as shown in Fig. 3. The $\chi$ of catecholamines like dopamine, noradrenaline and adrenaline is small, and their $\eta$ values are almost the same. It was found, moreover, that thylamine, amphetamine and phenetylamine also have a similar $r(\chi, \eta)$ as the $r(2.060,6.010)$ of dopamine. On the other hand, the $\eta$ of Glu 15, taurine $\left(\mathrm{NH}_{3}{ }^{+} \mathrm{CH}_{2} \mathrm{CH}_{2} \mathrm{SO}_{3}{ }^{-}\right)$9, Asp 4, and GABA 18 is larger than that of catecholamine analogs, but the $\chi$ is similar. Obviously, GABA and Glu analogs are chemically harder neurotransmitters than catecholamine analogs. This shows that serotonin $(\eta=5.570, \mathrm{~S}=0.179)$ and dopamine $(\eta=6.010, \mathrm{~S}=0.166)$ are softer than GABA $(\eta=8.165, \mathrm{~S}=0.122)$ and Glu $(\eta=7.245, \mathrm{~S}=0.138)$ in chemical structure. Here, the reciprocal of the hardness is the global softness (S), $\mathrm{S}=1 / \eta=(\partial N / \partial \mu)_{v(r)}{ }^{6,8)}$ Chemically soft dopamine and serotonin have higher polarizability than GABA and Glu and are easily oxidized. The electronic structure of 9 and guanidiylethansulfonic acid $(\mathrm{NH}=$ $\left.\mathrm{C}\left(\mathrm{NH}_{3}{ }^{+}\right) \mathrm{NHCH}_{2} \mathrm{CH}_{2} \mathrm{SO}_{3}^{-}\right) 19$ also are similar to those of GABA and Glu. These results indicate that the electronic structures of catecholamine and GABA analogs are controlled chemically by softness and bases and hardness and bases, respectively, while the ACh analogs are controlled by chemical hardness and acids.

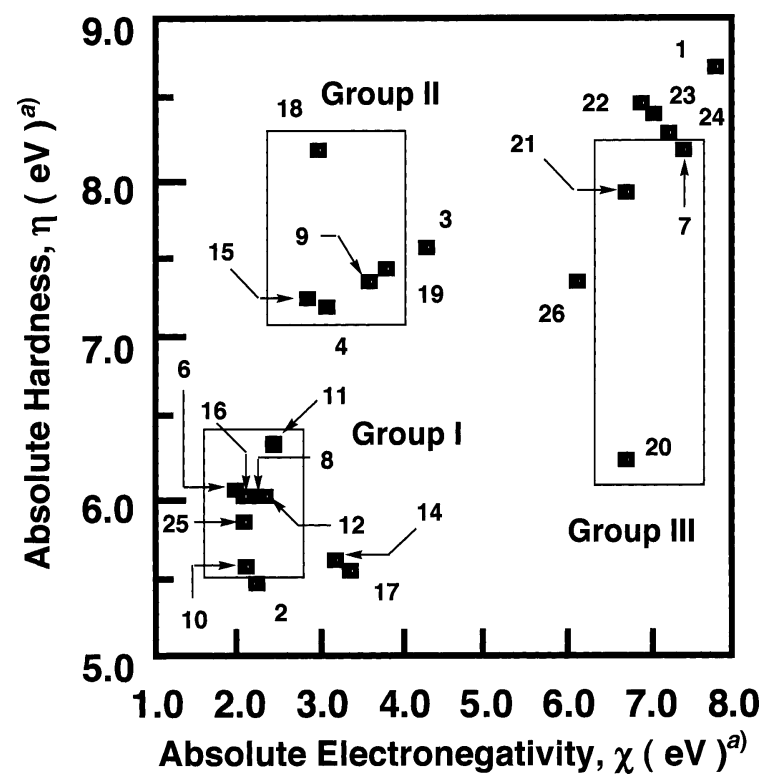

Fig. 3. Plot of a $\eta-\chi$ Diagram for the Relation of between Electronic Structure and Neurotransmitters

a) At the HF/6-31G** level. Numbers show the compounds listed in Table 1.

It is of interest, therefore, that based on electronic structure, neurotransmitters can be classified into three groups: catecholamine analogs (group I), GABA analogs (group II), and ACh analogs (group III), as shown in Fig. 3. This figure shows that compounds which have a similar electronic structure express similar activity. For example, the three ring anti-depressent imipramine $\mathbf{2 5} \boldsymbol{r}(2.070,5.840)$ is classified into group I and inhibits reuptake of dopamine $\mathbf{1 6}$ $\boldsymbol{r}(2.060,6.010)$, noradrenaline $\mathbf{1 2} \boldsymbol{r}(2.325,6.005)$, and adrenaline $6 r(1.980,6.040)$. The $\chi$ of 25 is equivalent to that of catecholamines but imipramine is obviously softer than catecholamines. This indicates that imipramine strongly interacts with the binding site of catecholamines.

Electronic Structure of the Central Nervous System in the Brain The nerve system is divided into the central nervous system (CNS) and the peripheral nervous system 

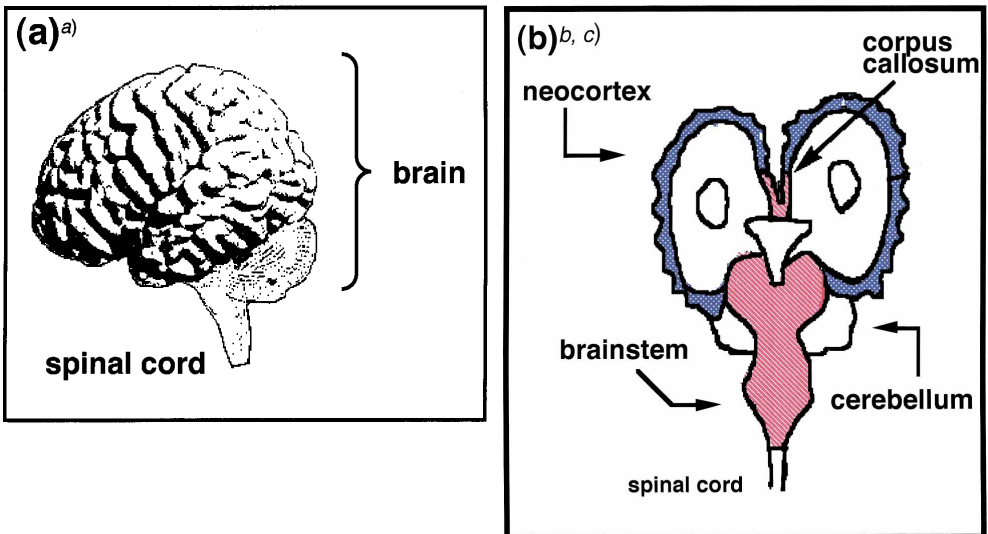

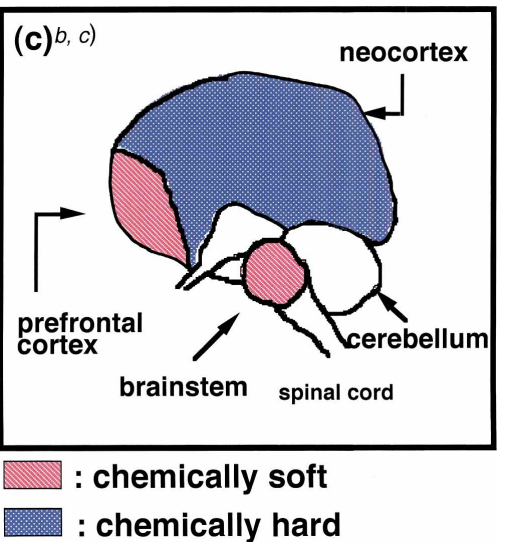

Fig. 4. Distribution of Electronic Structures in the Brain Obtained From Chemical Hardness

a) Structure of the central nervous system. Figure (a) was taken from http://material.miyazaki-c.ed.jp/cec/cg.seibutusikumi/sinkeikei/nou/a-831g2.jpg. b) Diagonal and dotted lines show the areas of the brain which are classified as chemically soft and hard, respectively. c) Left and right represents front (b) and flank (c), respectively.

Table 2. Calculated Absolute Hardness $(\eta)$ and Electronegativity $(\chi)$ of Optimized Neurotransmitters

\begin{tabular}{|c|c|c|c|c|}
\hline \multirow{2}{*}{ No. } & \multirow{2}{*}{ Compounds } & \multirow{2}{*}{$\begin{array}{l}\text { Relative molar ratio } \\
\text { vs. rectus abdominis } \\
\left.\text { of frog }{ }^{a}\right)\end{array}$} & $\begin{array}{c}\text { Absolute } \\
\text { electronegativity }\end{array}$ & \multirow{2}{*}{$\begin{array}{c}\text { Absolute hardness } \\
(\eta, \mathrm{eV})^{b)}\end{array}$} \\
\hline & & & $(\chi, \mathrm{eV})^{b)}$ & \\
\hline 22 & $\mathrm{CH}_{3} \mathrm{COOCH}_{2} \mathrm{CH}_{2} \mathrm{~N}^{+}\left(\mathrm{C}_{2} \mathrm{H}_{5}\right)_{3}$ & 5000 & 6.875 & 8.475 \\
\hline 23 & $\mathrm{CH}_{3} \mathrm{COOCH}_{2} \mathrm{CH}_{2} \mathrm{~N}^{+}(\mathrm{Me})\left(\mathrm{C}_{2} \mathrm{H}_{5}\right)_{2}$ & 300 & 7.050 & 8.410 \\
\hline 24 & $\mathrm{CH}_{3} \mathrm{COOCH}_{2} \mathrm{CH}_{2} \mathrm{~N}^{+}(\mathrm{Me})_{2}\left(\mathrm{C}_{2} \mathrm{H}_{5}\right)$ & 5 & 7.235 & 8.285 \\
\hline 7 & $\mathrm{CH}_{3} \mathrm{COOCH}_{2} \mathrm{CH}_{2} \mathrm{~N}^{+}(\mathrm{Me})_{3}$ & 1 & 7.415 & 8.175 \\
\hline
\end{tabular}

a) Taken from ref. 7. b) at ab initio $\mathrm{HF} / 6-31 \mathrm{G}^{* *}$ level.

(PNS). The CNS includes the brain and spinal cord, as shown in Fig. 4a. Here, our results show that the electronic structure of the brain can be depicted using the $\eta-\chi$ diagrams of the neurotransmitters. The monoamines dopamine, adrenaline, noradrenaline and serotonin, (the first three are known as catecholamines), are neurotransmitters of monoaminergic neurons. As is well known, the monoaminergic nerve pathways are systematically distributed in the nucleus of nerves of the brainstem. Our computed results show that serotonin and catecholamines are chemically softer than ACh and GABA. Moreover, these monoaminergic, such as dopaminergic, noradrenergic, and adrenergic, nerve systems are classified to the pathways, A8-A16, A1-A7, and $\mathrm{C} 1-\mathrm{C} 3$ (so-called A and $\mathrm{C}$ nerves), respectively, and they are placed around the brain and are unmyelinated nerves. In addition, the serotonergic nerve system is composed of the pathways, B1-B9. Then, the computed $r(\chi, \eta)$ values of dopamine, adrenaline, noradrenaline, and serotonin support that the $\mathrm{A}, \mathrm{B}$ and $\mathrm{C}$ nerves and brainstem are chemically soft. The prefrontal cortex also is chemically soft because an excess of chemically soft dopamine is secreted from the mesocortical dopaminergic A 10 nerve into the prefrontal cortex. However, GABA, Glu and taurine which act on the myelinated nerves, are widely distributed in the neocortex of the brain and are chemically hard, indicating that the neocortex is chemically hard.

The electronic structure of the brain can be divided based on hard and soft chemical properties as shown on the map. In Fig. 4, the diagonal lines show chemically soft and the dotted lines chemically hard areas. The results suggest that the function of the brain can also be divided using the electronic structures. Obviously, the brainstem and prefrontal cortex are chemically softer than the neocortex.

Correlation between the Activity and Chemical Hardness of Acetylcholine and Noradrenaline It has been reported that the relative molar ratio of equipotency for the activity of $\mathrm{ACh}$ derivatives against the rectus abdominis of flog increases in the following order; $\mathrm{AcOCH}_{2} \mathrm{CH}_{2} \mathrm{~N}^{+}(\mathrm{Et})_{3}$ $(5000)<\mathrm{AcOCH}_{2} \mathrm{CH}_{2} \mathrm{~N}^{+}(\mathrm{Me})(\mathrm{Et})_{2} \quad(300)<\mathrm{AcOCH}_{2} \mathrm{CH}_{2} \mathrm{~N}^{+}-$ $(\mathrm{Me})_{2}(\mathrm{Et})(5)<\mathrm{AcOCH}_{2} \mathrm{CH}_{2} \mathrm{~N}^{+}(\mathrm{Me})_{3}$ (1). ${ }^{7)}$ In the present study, bulky substituents of the ammonium cation at the $N$ atom played a dominant role in increasing the activity of the ACh agonist. It is suggested that there is no simple relationship between the structure and pharmacological activity of ACh derivatives. For this relationship, we found that the chemical hardness value $(\eta)$, which is a measure of the resistance to change in electron density, is directly proportional to the order of potency for the activity of ACh derivatives ( $R^{2}=0.963, R^{2}$ shows the correlation coefficient.). Moreover, the molar ratio of equipotency of these derivatives is inversely proportional to the absolute electronegativity $\left(R^{2}=\right.$ $0.977)$. These results show that the logarithmic molar ratios ( $\log$ (activity)) of equipotency of ACh derivatives correlated with the $\boldsymbol{r}(\chi, \eta)$ of the electronic structures (Table 2). Figure 5 shows a three dimensional plot (3D-diagram or 3D-scatter plot) of $\boldsymbol{r}(\chi, \eta)$ of ACh derivatives as the $x$ - and $y$-axis $v s . \log$ (activity for rectus abdominis of frog) as the $z$-axis.

Moreover, the activity for the regioselective effect of Me-substitution increases in the following order; $\mathrm{AcOCH}-$ $(\mathrm{Me}) \mathrm{CH}_{2} \mathrm{~N}^{+}(\mathrm{Me})_{3} \quad(100)<\mathrm{AcOCH}_{2} \mathrm{CH}(\mathrm{Me}) \mathrm{N}^{+}(\mathrm{Me})_{3} \quad$ (1). $\left.{ }^{7}\right)$ The regioselective effect for contraction of the rectus abdominis of frog of $\mathrm{AcOCH}(\mathrm{Me}) \mathrm{CH}_{2} \mathrm{~N}^{+}(\mathrm{Me})_{3}(\eta=5.215)$ and 


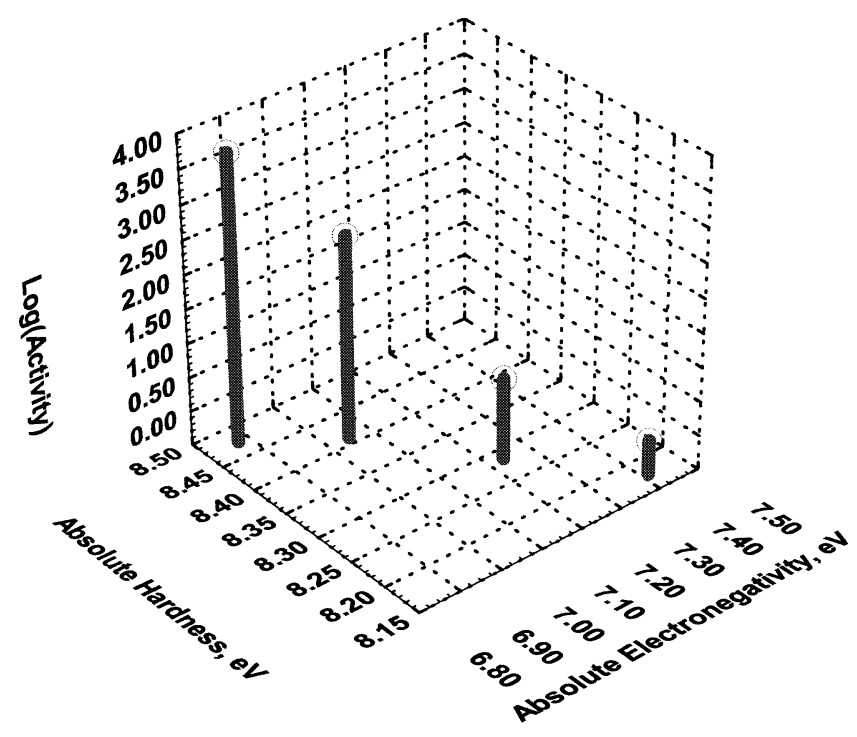

Fig. 5. 3D-Scatter Plot of Relationship between Bioactivity and Coordinate $\boldsymbol{r}(\chi, \eta)$ of the Electronic Structure of Acetylcholine Derivatives

Absolute electronegativity was used as the $x$-axis, absolute hardness as the $y$-axis, and $\log$ (activity) as the $z$-axis.

$\mathrm{AcOCH}_{2} \mathrm{CH}(\mathrm{Me}) \mathrm{N}^{+}(\mathrm{Me})_{3}(\eta=4.998)$ also shows a clear inverse linear correlation with the absolute hardness values. Finally, ACh is chemically softer and more acidic than the other ACh derivatives.

Agonists and Antagonists Figure 3 is a useful diagram with which to understand the relationships between agonists and antagonists for neurotransmitters. For instance, chlorpromazine $\mathbf{2}$ and haloperidol 14, major tranquilizers, are antagonists for dopamine. As their $\eta$ values are smaller than that of dopamine, $\mathbf{2}$ and $\mathbf{1 4}$ are chemically softer than dopamine. However, the differences, $\Delta \chi=\left|\chi_{\text {dopamine }}-\chi_{\text {chlorpromazine }}\right|$ (or $\left.\left|\chi_{\text {dopamine }}-\chi_{\text {haloperidol }}\right|\right)$, in the $\chi$ values are large. This suggests that the antagonist has a similar $\chi$ value, and the difference in $\eta$ is large. On the other hand, the gap in the $\eta$ value between imipramine and dopamine is smaller than that $\left(\mid \eta_{\text {dopamine }}-\right.$ $\left.\eta_{\text {chlorpromazine }} \mid\right)$ between dopamine and chlorpromazine, therefore, compound $\mathbf{2 5}$ has a dopamine-like effect. The idea can also be applied to diazepam 17. Although the gap in the $\chi$ value between compounds $\mathbf{1 7}$ and $\mathbf{1 8}$ is nearly $\sim 0$, the gap for $\left|\eta_{\mathrm{GABA}}-\eta_{\text {diazepam }}\right|$ of $\eta$ values is about $2.6(\mathrm{eV})$. This means that diazepam is an antagonist of GABA (see Fig. 3).

The electronic structure coordinate of oxisotoremorine $\mathbf{2 6}$ is $r(6.110,7.360)$, and it is distributed in the area of acetylcholine (group III). In the $\eta-\chi$ activity diagram, clearly, oxisotoremorine is harder than nicotine $\mathbf{2 0}$ since the $\eta$ value of $\mathbf{2 6}$ is larger than that of $\mathbf{2 0}$. This supports that compound $\mathbf{2 6}$ is muscarine agonist for $\mathrm{ACh} \mathbf{7}$, in fact, compound $\mathbf{2 6}$ is strongly a muscarine agonist. ${ }^{15)}$ According to our method, two pharmacologically distinguishable types, musucarine and nicotine, of 7 in the cholinergic system are different in their chemical hardness, and the differences of hardness of 7 are caused by variable conformation of 7 . However, the gap of total energy of cisoid and transoid in 7 is nearly $\sim 0 \mathrm{kcal} / \mathrm{mol}$ as described above (see section "Structure and Chemical Hardness and $\eta-\chi$ Activity Diagram of Central Neurotransmitters"). Moreover, the absolute electronegativity $(\chi)$ value of the cholinergic drugs $\mathbf{1}, \mathbf{7}$, and 26, interestingly, is larger than dopaminergic and adrenergic drugs. Imipramine 25, flu-

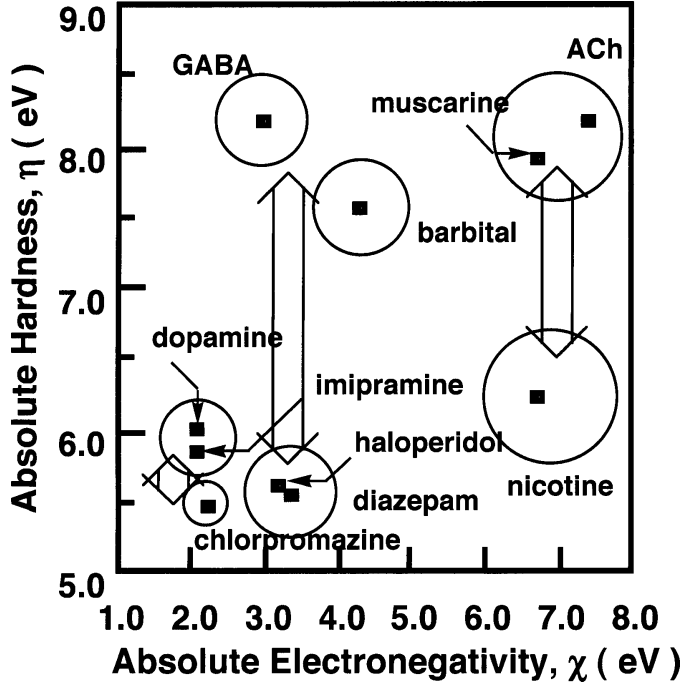

Fig. 6. Relationship of Electronic Structures between Agonists and Antagonists for Neurotransmitters

Arrows indicate the compounds with a relationship between agonists and antagonists. The compounds in circles are agonists. For example, imipramine is an agonist of dopamine.

oxetine 27, and milnacipran $\mathbf{2 8}$ which are therapeutic drugs for depression, have similar $\eta$ values, and their electronic structure belongs to group I. Finally, the relationship between agonists and antagonists with the neurotransmitters can be derived and is shown in Fig. 6.

\section{Discussion}

In this study, we showed that $\eta-\chi$ activity diagrams plotted the coordinates of electronic structures of neurotransmitters like dopamine, catecholamines and ACh. From the 2-D diagram (see Fig. 3), it is obvious that the electronic structures of neurotransmitters can be classified into three groups. Dopa, dopamine, and noradrenaline belong to group I. GABA, Asp, taurine, and Glu are distributed in group II. $\mathrm{ACh}$, nicotine and muscarine belong to group III. Therefore, the differences in the electronic structures of neurotransmitters are obvious. Although the absolute electronegativity $\chi$ of dopamine $\mathbf{1 6}$ is about equal to that of chlorpromazine $\mathbf{2}$, the absolute hardness $\eta$ is larger than that of 2 . Therefore, the two-dimensional coordinates of compounds 2 and $\mathbf{1 6}$ could account for the difference in chemical properties. The softer chlorpromazine $\mathbf{2}$ binds more tightly with the dopamine receptor (DR) than does dopamine itself. The stabilization energy $(\Delta E)$ for the interaction between chlorpromazine and DR is given by Eq. 5. ${ }^{16)}$

$$
\Delta E=-\left(\chi_{\text {dopamine }}-\chi_{\mathrm{DR}}\right)^{2} / 4\left(\eta_{\text {dopamine }}+\eta_{\mathrm{DR}}\right)
$$

In Eq. 5, $\Delta E$ increases when the denominator decreases to less than $\left(\chi_{\text {dopamine }}-\chi_{\mathrm{DR}}\right)^{2}$ value, namely when the $y$-axis (absolute hardness $\eta$ ) values decrease, as shown in Fig. 3.

The $\eta-\chi$ activity diagram provides important information on the relation between agonist and antagonist for neurotransmitters. Chlorpromazine and diazepam 17 are antagonists for dopamine and GABA, respectively. For instance, he gaps in electronic structure coordinate $r(\chi, \eta)$ for chlorpromazine $r\left(\chi_{\text {chlorpromazine }}, \eta_{\text {chlorpromazine }}\right)$ and dopamine $\boldsymbol{r}\left(\chi_{\text {dopamine }}, \eta_{\text {dopamine }}\right)$ are equal to $\Delta \chi=\chi_{\text {chlorpromazine }}-\chi_{\text {dopamine }}$ $(=0.18)$ and $\Delta \eta=\eta_{\text {chlorpromazine }}-\eta_{\text {dopamine }}(=0.55)$, respec- 


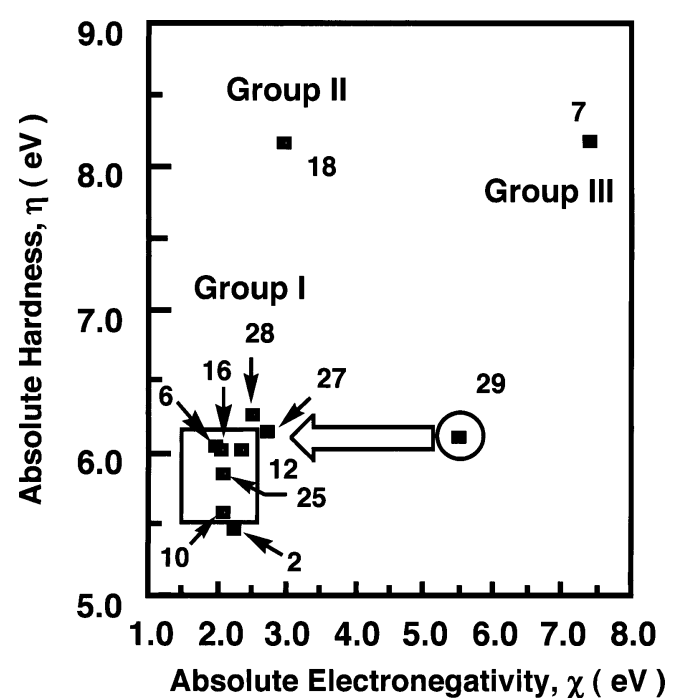

Fig. 7. Design of Anti-depressant Drugs

Compound 29 is not an anti-depressant. In order to design anti-depressants, conditions like $\eta_{\text {selotonin(or dopamine) }}-\eta_{\text {anti-depressant }}<0$ and $\chi_{\text {selotonin(or dopamine) }}-\chi_{\text {anti-depressant }} \sim 0$ must be satisfied. That is, anti-depressants are chemically harder than selotonin and dopamine. However, it seems probable that anti-maniac drugs are chemically softer than selotonin.

tively. For GABA, similarly, the gaps of $\boldsymbol{r}(\chi, \eta)$ for diazepam $\boldsymbol{r}\left(\chi_{\text {diazepam }}, \eta_{\text {diazepam }}\right)$ and GABA $r\left(\chi_{\mathrm{GABA}}, \eta_{\mathrm{GABA}}\right)$ are $\Delta \chi=\chi_{\text {di- }}$ ${ }_{\text {azepam }}-\chi_{\mathrm{GABA}}(=0.40)$ and $\Delta \eta=\eta_{\text {diazepam }}-\eta_{\mathrm{GABA}}(=2.62)$. The results suggest that the gap $\Delta r(\Delta \Delta \chi, \Delta \Delta \eta)$ in electronic structure of antagonist with agonist is equal to $\Delta \Delta \chi \fallingdotseq 0$ and $\Delta \Delta \eta=\left|\eta_{\text {antagonist }}-\eta_{\text {agonist }}\right|>0$. Thus, it is indicated that $\Delta \Delta \chi=\left|\chi_{\text {antagonist }}-\chi_{\text {agonist }}\right|=0$ and $\Delta \Delta \eta=\left|\eta_{\text {antagonist }}-\eta_{\text {agonist }}\right|>0$ is an essential condition to design an antagonist against an agonist.

The coordinate $r(\chi, \eta)$ would be a useful tool with which to investigate the electronic structure of the brain in the central nervous system. GABA, Gly, Asp, or Glu acts on the marrow nerve system, while catechol amines like dopamine and serotonin act on the non-marrow nerve system in the brain. From the results, the electronic structure of the brain can be described by the map shown in Fig. 4. The dopaminergic, adrenergic, noradrenergic, and serotonergic nerve systems, brainstem, and corpus callosum belong to group I, however, the neocortex belongs to group II. As the electronic structures of the amine nerve system like dopamine, epinephrine, and serotonin have small $\chi$ and $\eta$ values, these systems are chemically soft. However, GABA and ACh nerve systems have a large $\eta$ value and are chemically harder than the amine nerve system. In addition, the prefrontal cortex which is rich in dopamine is chemically soft.

The analysis of structure-activity relationships using $\eta-\chi$ activity diagrams may be qualitative, but the method is useful for estimating the biological properties of chemicals. What are the coordinates of antidepressants like imipramine 25, fluoxetine $\mathbf{2 7}$ and milnacipran $\mathbf{2 8}$, in the $\eta-\chi$ activity diagram (see Fig. 3)? The results are shown in Fig. 7. Compounds $\mathbf{2 5}, \mathbf{2 7}$, and $\mathbf{2 8}$ have similar electronic structures to agonists like dopamine, adrenaline, and noradrenaline. It is clear that antidepressants do not have an electronic structure $r\left(\chi_{29}, \eta_{29}\right)$ like the unknown compound 29. Essential for the electronic structure $r\left(\chi_{\text {antidepressant }}, \eta_{\text {antidepressant }}\right)$ as an antidepressant is $\chi_{\text {antidepressant }}=\left|\chi_{\text {dopamine }}-\chi_{29}\right| \fallingdotseq 0$ and $\eta_{\text {antidepressant }}=$ $\left|\eta_{\text {dopamine }}-\eta_{29}\right| \fallingdotseq 0$, so when compound 29 has $\chi_{\text {antidepressant }}=$ $\left|\chi_{\text {dopamine }}-\chi_{29}\right| \gg 0$, it probably does not act as an antidepressant. Then, if compound $\mathbf{2 9}$ is to be used as a dopamine agonist or antidepressant, the target has to be designed so as to satisfy the condition, $\eta_{\text {antidepressant }}=\left|\eta_{\text {dopamine }}-\eta_{29}\right| \fallingdotseq 0$ and $\chi_{\text {antidepressant }}=\left|\chi_{\text {dopamine }}-\chi_{29}\right| \fallingdotseq 0$.

Finally, the following rules can be derived

$$
\begin{aligned}
\text { Condition 1: } & \Delta \chi=\mid \chi_{\mathrm{L}}^{0}-\chi_{\text {agonist }}=\sim 0, \eta_{\mathrm{L}}^{0} \sim \eta_{\text {agonist. }} \\
& \text { an agonist }\left(\chi_{\mathrm{a}}, \eta_{\mathrm{a}}\right) \text { has an electronic struc- } \\
& \text { ture's coordinate } r(\chi, \eta) \text { similar to a ligand } \\
& \boldsymbol{r}\left(\chi_{\mathrm{L}}^{0}, \eta_{\mathrm{a}}\right) . \\
\text { Condition 2: } & \Delta \chi=\left|\chi_{\mathrm{L}}^{0}-\chi_{\text {antagonist }}\right|=\sim 0, \quad \eta_{\mathrm{L}}^{0}>\eta_{\text {antagonist }} \\
& \left(\text { or } \quad \eta_{\mathrm{L}}^{0}<\eta_{\text {antagonist }}\right) .
\end{aligned}
$$

These conditions are essential for the antagonist of the ligand. Further, if a target compound (x) is neither an agonist nor an antagonist for the ligand, then

Condition 3: $\Delta \chi=\left|\chi_{\mathrm{L}}^{0}-\chi_{\mathrm{x}}\right|>0$, $\Delta \eta=\left|\eta_{\mathrm{L}}^{0}-\eta_{\mathrm{x}}\right|>0$.

\section{Conclusion}

We have shown here that the present ab initio MO calculations of the electronic structures of neurotransmitters provide three groups, I, II, and III, in the $\eta-\chi$ diagram. The catechol amines like dopamine, adrenaline, and noradrenaline are distributed in group I, whereas GABA, tauline, and Glu, belong to group II. The results indicate that the coordinate $r(\chi, \eta)$ of the electronic structure of a neurotransmitter plays an important role in the activity of the neurotransmitter in the central nervous system. Although differences in the activity of muscarine and nicotine as acetylcholine agonists are not known, that nicotine $\boldsymbol{r}(6.700,6.240)$ is chemically softer than muscarine $r(6.715,7.915)$ suggests that the activity of acetylcholine agonists is related to the $r(\chi, \eta)$ of the ammonium salt.

According to the $\eta-\chi$ diagram, the electronic structure of the brain can be qualitatively represented with a distribution map using the $\eta$ and $\chi$ of neurotransmitters. Although the neocortex is chemically hard, brainstem and A, B, and C nerve systems are chemically very soft. Imipramine and fluoxetine which are chemically soft thus belong to group I, and have been used in the treatment of anixety and depression, and it is suggested that these drugs are transported to chemically soft brainstem and A, B, and C nerve systems (see Fig. 4). Our study clearly showed that coordinate $r(\chi, \eta)$ of the electronic structure plays an important role in the expression of biological functions. It is of pharmacological importance whether the $\boldsymbol{r}(\chi, \eta)$ of drug belongs to group I, II, or III in the brain.

\section{References and Notes}

1) Kobayashi S., Hamashima H., Kurihara M., Miyata N., Tanaka A., Chem. Pharm. Bull., 46, 1108-1115 (1998).

2) Kobayashi S., Sugaya T., Sakata N., Uebayashi M., Sameshima K., Tanaka A., Chem. Pharm. Bull., 49, 1230-1240 (2001).

3) Peason R. G., Songstand J., J. Am. Chem. Soc., 89, 1827-1836 (1967).

4) Parr R. G., Donnelly R. A., Palke W. E., J. Chem. Phys., 68, 38013807 (1978).

5) Shanker S., Parr R. G., Proc. Natl. Acad. Sci. U.S.A., 82, 264-266 (1985). 
6) Yang W., Parr R. G., Proc. Natl. Acad. Sci. U.S.A., 82, 6723-6726 (1985).

7) Richards W. G., "Quantum Pharmacology," Butterworth \& Co. (Publishers) Ltd., London, 1983.

8) Fluoxetine and milnacipran are selective serotonin reuptake inhibitor (SSRI) and serotonin-noradrenalin reuptake inhibitor (SNRI), respectively: Wong D. T., Horng J. S., Bymaster F. P., Hauser K. L., Molloy B. B., Life Sci., 15, 471- 479 (1975).

9) Gaussian 98 (Gaussian, Inc., U.S.A.) and Spartan 02 (Wavefunction, Inc., U.S.A.), a molecular orbital software package, respectivly. We calculated on the Compac DS10 and Windows (Pentium 4) machine.

10) Parr R. G., Pearson R. G., J. Am. Chem. Soc., 105, 7512-7516 (1983).

11) Pullman B., Berthod H., Gresh N., C R Acad. Sci. Hebd. Seances Acad. Sci. D., 280, 1741-1744 (1975).

12) Jellinek F., Acta Crystallogr., 10, 277-281 (1957).

13) Chothia C., Pauling L., Nature (London), 219, 1156-1157 (1968).

14) Kier L. B., "Fundamental Concepts in Drug-Receptor Interactions," Academic Press, New York, 1970.

15) Kier L. B., J. Pharm. Sci., 59, 112-114 (1970).

16) Alonso J. A., Baekelandt B. G., Balbas L. C., Chattaraji P. K., Gazquez J. L., Grice M. E., Komorowski L., March N. H., Motier W. J., Murray J. S., Nalcwajiski R. F., Parr R. G., Peason R. G., Politzer P.,
Schoonheyte R. A., Sen K. D., "Structure and Bonding," Vol. 80, Springer-Verlag, Berlin, 1993.

Abbreviations: Choline; 2-hydroxy- $N, N, N$-trimethylammonium, Chlorpromazine; 2-chloro-10-(3-dimethylaminopropyl) phenothiazine, Barbital; 5,5diethyl-2,4,6(1H,3H,5H)-pyrimidinetrione, Asp; aspartic acid, Amphetamine; 2-phenyl-1-methylethaneamine, Adrenaline; $(R)-1-\left(3^{\prime}, 4^{\prime}\right.$-dihydroxyphenyl)2-methylamino)-ethanol, Acetylcholine; 2-acetoxy- $N, N, N$-trimethylammonium, Thyramine; 2-(4'-hydroxyphenyl)-ethaneamine, Taurine; 3-aminoethylsulfonic acid, Serotonin; 3-( $\beta$-aminoethyl)-5-hydroxyindole, Phenethyl amine; 1-amino-2-phenylethane, Noradrenaline; 1-(3,4-dihydroxyphenyl)-2aminoethanol, $m$-Thyramine; 2-(3'-hydroxyphenyl)-ethaneamine, Haloperidol; 4-[4-( $p$-chlorophenyl)-4-hydroxypiperidino]-4'-fluorobutyrophenone, Glu; glutamic acid, Dopamine; 2-(3',4'-dihydroxyphenyl)-ethylamine, Diazepam; 7-chloro-1,3-dihydro-1-methyl-5-phenyl-2H-1,4-benzodiazepin2-one, GABA; $\gamma$-aminobutanoic acid; Guanidiyltaurine; $N$-guanidylethylsulfonic acid, Nicotine; 1-methyl-2-(3-pyridyl)pyrrolidinyl)pyridine, Mus-

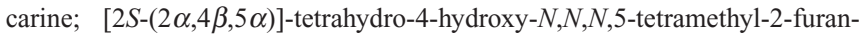
methanaminium, Imipramine; 5-(3-dimethylaminopropyl)-10,11-dihydro-5 $\mathrm{H}$ dibenz[ $b$ f $]$ azepine, Oxotremorine; 1-[4-(1-pyrrolidinyl)-2-butynyl]-2-pyrrolidinone, Fluoxetine; $N$-methyl-3-( $p$-trifluoromethylphenoxy)-3-phenylpropylamine, Milnacipran; (1S,2R)-1-phenyl-2-[(R)-1-amino-2-propynyl]- $N, N$-diethylcyclopropanecarboxamide. 